\title{
A stochastic process model for resistance deterioration of aging bridges
}

Cao Wang (D)

\section{Correspondence:}

cao.wang@monash.edu

Department of Civil Engineering,

Monash University, 3800 Clayton,

Australia

\begin{abstract}
The serviceability and safety level of bridges often deteriorates due to the environmental or operational conditions. A stochastic deterioration model is of essential importance for describing the time-variation of bridge performance (e.g., stiffness and strength), and for use in the estimate of bridge reliability under a probabilistic framework. In this context, the Gamma process has been widely used to model the resistance deterioration of aging bridges, yet suffers from the deficiency that the statistical characteristics of the process (namely mean value, variance and autocorrelation) are not mutually independent. This paper presents a new stochastic model for bridge resistance deterioration. As a modified version of the Gamma process-based one, the proposed model includes a new parameter that can release the dependence of the process autocorrelation and variance on the mean value. The impact of the new deterioration model on the time-dependent reliability of aging bridges is studied.
\end{abstract}

Keywords: Resistance deterioration, Stochastic process, Aging bridges, Structural reliability

\section{Introduction}

In-service bridges often suffer from the deterioration of performance (e.g., stiffness and strength) subjected to aggressive environmental or operational conditions, which may further decline the bridge safety level below the baseline as assumed in design. It is of essential importance to model the deterioration process of aging bridges reasonably for use in the estimate of structural service reliability, providing a decision-aid tool for maintenance and repair measures for the bridges (Mori and Ellingwood 1993; Enright and Frangopol 1998; Stewart and Val 1999; OBrien et al. 2014).

The Gamma process has been widely used to describe the resistance deterioration (the difference between the initial and the degraded resistances) of aging bridges (Dieulle et al. 2003; Saassouh et al. 2007; van Noortwijk et al. 2007; Li et al. 2015; Wang et al. 2015; Wang et al. 2019), which is, by nature, a non-decreasing process and enables the autocorrelation of the resistance deterioration process on the temporal scale to be considered. The monotonicity of a Gamma process is guaranteed by its nonnegative and independent increments. These increments also follow a Gamma distribution, and have an identical

(c) The Author(s). 2020 Open Access This article is licensed under a Creative Commons Attribution 4.0 International License, which permits use, sharing, adaptation, distribution and reproduction in any medium or format, as long as you give appropriate credit to the original author(s) and the source, provide a link to the Creative Commons licence, and indicate if changes were made. The images or other third party material in this article are included in the article's Creative Commons licence, unless indicated otherwise in a credit line to the material. If material is not included in the article's Creative Commons licence and your intended use is not permitted by statutory regulation or exceeds the permitted use, you will need to obtain permission directly from the copyright holder. To view a copy of this licence, visit http://creativecommons.org/licenses/by/4.0/. 
scale parameter (Kahle et al. 2016). This fact, unfortunately, indicates that both the autocorrelation and the variance of a Gamma process are dependent on the mean value of the process. More precisely, once the mean of a Gamma process is known, then the autocorrelation and the overall shape of the variance would be fully determined accordingly. Such dependence may halter the applicability of a Gamma process-based deterioration model in structural reliability assessment, especially for the cases where observed data are available to calibrate the unknown parameters in the deterioration process.

Motivated by this, a new stochastic deterioration model is presented in this paper, which introduces an additional parameter that releases the dependence of the process autocorrelation and variance on the mean value. The characteristics of the proposed model are discussed, and the impact of the deterioration model on the time-dependent reliability of aging bridges is investigated.

\section{Stochastic model for resistance deterioration}

In the presence of structural resistance deterioration, the degraded resistance at time $t$, $R(t)$, can be expressed as follows,

$$
R(t)=R_{0} \cdot G(t)
$$

where $R_{0}$ is the initial resistance and $G(t)$ is the deterioration function (a stochastic process). For a reference period of $(0, t)$, in order to reflect the randomness nature of resistance deterioration, the period $(0, t)$ is subdivided into $n$ identical sections, namely $\left(t_{0}=0, t_{1}\right),\left(t_{1}, t_{2}\right), \ldots\left(t_{n-1}, t_{n}=t\right)$. With this, the deterioration function valued at time $t_{n}, G\left(t_{n}\right)$, is Li et al. (2015).

$$
G\left(t_{n}\right)=1-\sum_{i=1}^{n} \hat{d}\left(t_{i}\right) \cdot \epsilon\left(t_{i}\right)
$$

where $\hat{d}\left(t_{i}\right)$ is the mean degradation within $\left(t_{i-1}, t_{i}\right)$, which is deterministic, and $\epsilon\left(t_{i}\right)$ is a sequence of independent random variables associated with each interval. When a Gamma process is employed to describe the resistance deterioration, the noise item $\epsilon\left(t_{i}\right)$ in Eq. (2) would follow a Gamma distribution, having a mean value of 1 and a standard deviation of $\sqrt{\frac{\xi}{\hat{d}\left(t_{i}\right)}}$, where $\xi$ is a constant defining the uncertainty associated with $G(t)$. This implies that the overall shape of the variance of the deterioration function is fully dependent on the mean deterioration function, which is untenable in many cases.

Mathematically, the item $\epsilon\left(t_{i}\right)$ in Eq. (2) should be positive due to the physical constraints of a deterioration process. Note, also, that the mean value of $\epsilon\left(t_{i}\right)$ equals 1 since $\hat{d}\left(t_{i}\right)$ defines the shape of degradation. Let $\mu_{\bullet}$ and $\sigma_{\bullet}$ denote the mean value and standard deviation of the variable in subscript respectively. With this, under the assumption of independent increments of the deterioration process, it follows,

$$
\mu_{G\left(t_{n}\right)}=1-\sum_{i=1}^{n} \hat{d}\left(t_{i}\right)
$$

and

$$
\sigma_{G\left(t_{n}\right)}=\sqrt{\sum_{i=1}^{n}\left(\hat{d}\left(t_{i}\right) \cdot \sigma_{\epsilon\left(t_{i}\right)}\right)^{2}}
$$

In this paper, it is proposed that $\hat{d}\left(t_{i}\right)$ and $\epsilon\left(t_{i}\right)$ satisfy,

$$
\hat{d}\left(t_{i}\right)=k \cdot t_{i}^{m} \cdot\left(t_{i}-t_{i-1}\right)
$$




$$
\sigma_{\epsilon\left(t_{i}\right)}=\frac{p \cdot t_{i}^{q}}{\sqrt{t_{i}-t_{i-1}}}
$$

where $k, m, p$ and $q$ are four time-invariant parameters defining the scale and shape of $\hat{d}\left(t_{i}\right)$ and $\epsilon\left(t_{i}\right)$. Substituting Eqs. (5) and (6) into Eqs. (3) and (4), as $n \rightarrow \infty$, one has,

$$
\mu_{G(t)}=1-\frac{k}{m+1} t^{m+1}
$$

and

$$
\sigma_{G(t)}=\frac{k p}{\sqrt{2 m+2 q+1}} t^{m+q+0.5}
$$

Note that the parameter $q$ in Eqs. (6) and (8) equals $-0.5 m$ if employing a Gamma process, with which the shape of $\sigma_{G(t)}$ would be dependent on $\mu_{G(t)}$, yielding a redundant constraint for the relationship between $\sigma_{G(t)}$ and $\mu_{G(t)}$. Eq. 8 also implies that the proposed deterioration model is a generalized form of a Gamma process.

With Eq. (8), the correlation coefficient of $G\left(t_{l}\right)$ and $G\left(t_{j}\right)(1 \leq j \leq l \leq n)$, $\rho_{l, j}$, is determined by

$$
\rho_{l, j}=\frac{\sigma_{G\left(t_{j}\right)}}{\sigma_{G\left(t_{l}\right)}}=\left(\frac{t_{j}}{t_{l}}\right)^{m+q+0.5}
$$

Eq. 9 implies that the autocorrelation coefficient of resistance deterioration is affected by both the shape of mean resistance degradation (c.f. the parameter $m$ ) and the variance of resistance deterioration (c.f. the parameter $q$ ).

There are totally four parameters involved in the proposed deterioration model, namely $k, m, p$ and $q$. These parameters can be calibrated if the observed data for the resistance deterioration are available. First, by fitting the overall trend (mean value) of the deterioration process with respect to time $t$ with the method of least squares, the parameter $m$ is determined by the shape of the time-variant mean deterioration according to Eq. (7). For example, a linear deterioration shape gives $m=0$ while a parabolic deterioration shape results in $m=1$. Simultaneously, the parameter $k$ is obtained by the least squares regression. Similarly, the remaining parameters $p$ and $q$ can be calibrated based on the time-variant standard deviation of the resistance deterioration (c.f. Eq. (8)).

The item $\epsilon\left(t_{i}\right)$ in Eq. (2) could be modeled as a Gamma, uniform, Beta or lognormal random variable, among other distribution types for positive variables, depending on the specific problems, considering the fact that $\epsilon\left(t_{i}\right)$ is a positive variable by nature. For illustration purpose, Figs. $1 \mathrm{a}$ and b plots some sampled trajectories of resistance deterioration for a reference period of 40 years. It is assumed that $m=0.5, k=0.0018$ year, and $t_{i}-t_{i-1}=0.1$ year, so that the deterioration function valued at the end of 40 years has a mean value of 0.7 . It is also assumed that $\sigma_{G(40)}=0.14$ with a Gamma distribution for each $\epsilon\left(t_{i}\right)$. The parameter $q$ is 0.5 in Fig. 1a and 1.5 in Fig. 1b, with which the remaining parameter $p$ in Eq. (8) can be calculated uniquely. The comparison between Figs. 1a and b shows that, when the variance of $G(40)$ is fixed, a smaller value of $q$ results in a greater variability of the deterioration process. This is explained by Fig. 1c, where the time-variant standard deviations of the deterioration function are calculated with Eq. (8) and are plotted for different values of $q$. By noting that $q=-0.5 m=-0.25$ if using a Gamma process, it is observed that a Gamma process-based deterioration model overestimates the variance of deterioration function if $q>-0.5 m$ and underestimates the 


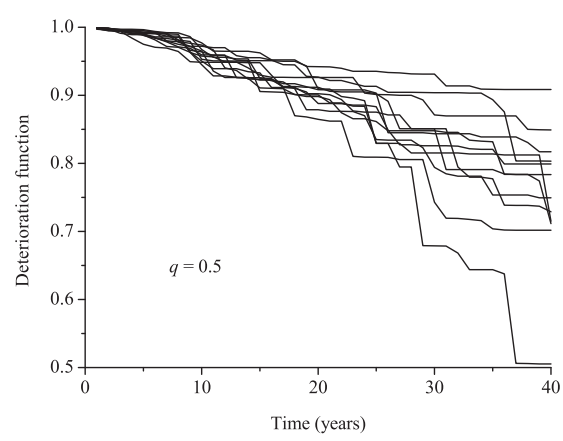

(a)

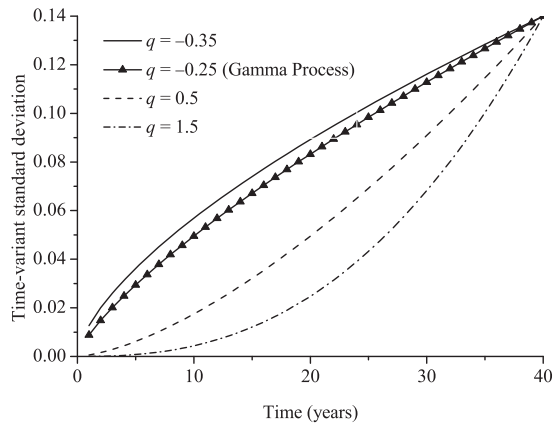

(c)

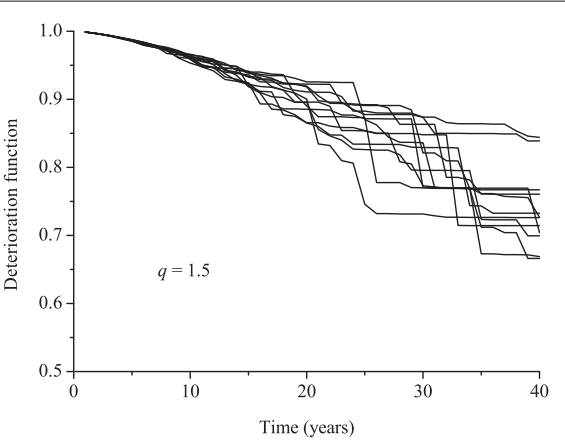

(b)

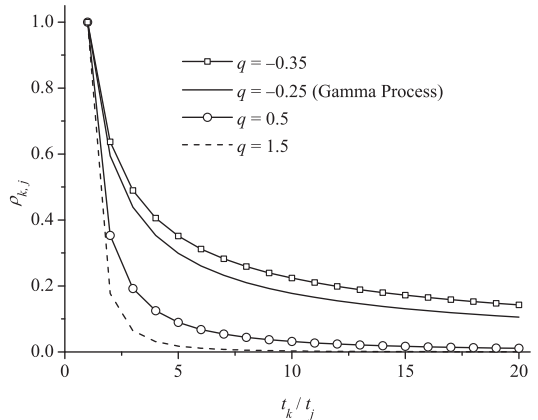

(d)

Fig. 1 Illustration of the proposed resistance deterioration model. $\mathbf{a}$ and $\mathbf{b}$ Sampled deterioration trajectories; c Time-variant standard deviation with different values of $q$; $\mathbf{d}$ Autocorrelation coefficient with different values of $q$

variance if $q<-0.5 \mathrm{~m}$. Figure $1 \mathrm{~d}$ presents the dependence of the autocorrelation coefficient on the time lag. It is seen that a larger value of $q$ leads to a smaller autocorrelation in resistance deterioration.

Finally, the convergence of the proposed deterioration model is discussed. The term convergence herein means that the probabilistic behaviour of the deterioration model as defined in Eqs. (5) and (6) does not depend on the choice of $t_{i}-t_{i-1}$, provided that $t_{i}-t_{i-1} \rightarrow 0$. Consider the characteristic function of $G\left(t_{n}\right)$ in Eq. (2), which, if exists, can uniquely determine the probability distribution function of $G\left(t_{n}\right)$ and vice versa (Billingsley 1986). Illustratively, assume that $\epsilon\left(t_{i}\right)$ follows a Gamma distribution with a mean value of 1 and a standard deviation given in Eq. (6). Let $a_{d \epsilon, i}$ and $b_{d \epsilon, i}$ denote the shape parameter and scale parameter of $\hat{d}\left(t_{i}\right) \cdot \epsilon\left(t_{i}\right)$ respectively, with which

$$
b_{d \epsilon, i}=\frac{\sigma_{\hat{d}\left(t_{i}\right) \cdot \epsilon\left(t_{i}\right)}^{2}}{\mu_{\hat{d}\left(t_{i}\right) \cdot \epsilon\left(t_{i}\right)}}=k p^{2} t_{i}^{m+2 q}
$$

and

$$
a_{d \epsilon, i}=\frac{\mu_{\hat{d}\left(t_{i}\right) \cdot \epsilon\left(t_{i}\right)}}{b_{d \epsilon, i}}=p^{-2} t_{i}^{-2 q}\left(t_{i}-t_{i-1}\right)
$$


Let $\phi_{X}(\mathrm{i} \tau)$ denote the characteristic function of random variable $X$, where $\mathrm{i}=\sqrt{-1}$ is the imaginary unit. Since $\hat{d}\left(t_{i}\right) \cdot \epsilon\left(t_{i}\right)$ is Gamma distributed,

$$
\phi_{\hat{d}\left(t_{i}\right) \cdot \epsilon\left(t_{i}\right)}(\mathrm{i} \tau)=\left(\frac{1}{1-b_{d \epsilon, i} i \tau}\right)^{a_{d \epsilon, i}}=\left(\frac{1}{1-k p^{2} t_{i}^{m+2 q} \cdot \mathrm{i} \tau}\right)^{p^{-2} t_{i}^{-2 q}\left(t_{i}-t_{i-1}\right)}
$$

By noting the independent increments of the deterioration process, the characteristic function of $1-G\left(t_{n}\right)$ is given by

$$
\begin{aligned}
\phi_{1-G\left(t_{n}\right)}(\mathrm{i} \tau) & =\prod_{i=1}^{n} \phi_{\hat{d}\left(t_{i}\right) \cdot \epsilon\left(t_{i}\right)}(\mathrm{i} \tau)=\exp \left(\sum_{i=1}^{n} \ln \phi_{\hat{d}\left(t_{i}\right) \cdot \epsilon\left(t_{i}\right)}(\mathrm{i} \tau)\right) \\
& =\exp \left(-\sum_{i=1}^{n} p^{-2} t_{i}^{-2 q}\left(t_{i}-t_{i-1}\right) \cdot \ln \left(1-k p^{2} t_{i}^{m+2 q} \cdot \mathrm{i} \tau\right)\right) \\
& =\exp \left(-p^{-2} \int_{0}^{t} x^{-2 q} \ln \left(1-k p^{2} x^{m+2 q} \cdot \mathrm{i} \tau\right) \mathrm{d} x\right)
\end{aligned}
$$

Eq. 13 implies that the characteristic function of $1-G\left(t_{n}\right)$ (and thus $\left.G\left(t_{n}\right)\right)$ is independent of the absolute value of $t_{i}-t_{i-1}$ provided that $t_{i}-t_{i-1} \rightarrow 0$. Specifically, if $q=-0.5 \mathrm{~m}$ in Eq. (13), then

$$
\begin{aligned}
\phi_{1-G\left(t_{n}\right)}(\mathrm{i} \tau) & =\exp \left(-p^{-2} \ln \left(1-k p^{2} \cdot \mathrm{i} \tau\right) \int_{0}^{t} x^{-2 q} \mathrm{~d} x\right) \\
& =\exp \left(-p^{-2} \ln \left(1-k p^{2} \cdot \mathrm{i} \tau\right) \cdot \frac{t^{1-2 q}}{1-2 q}\right) \\
& =\left(\frac{1}{1-k p^{2} \cdot \mathrm{i} \tau}\right)^{\frac{t^{m+1}}{p^{2}(m+1)}}
\end{aligned}
$$

which is the characteristic function of a Gamma distribution with a shape parameter of

$\frac{t^{m+1}}{p^{2}(m+1)}$ and a scale parameter of $k p^{2}$, consistent with the case of a Gamma process-based deterioration model.

\section{Time-dependent reliability assessment}

For a bridge with a time-variant resistance $R(t)$, let $S(t)$ be the load effect at time $t$. The bridge is deemed to be safe within a reference period of [0,T] if $R(t) \geq S(t)$ holds for $\forall t \in$ $[0, T]$. Correspondingly, the time-dependent reliability, denoted by $\mathbb{L}(T)$, is estimated by

$$
\mathbb{L}(T)=\operatorname{Pr}\{R(t) \geq S(t), \forall t \in[0, T]\}
$$

where $\operatorname{Pr}()$ is the probability of the event in the bracket.

A Poisson process can be used to model the occurrence of extreme load events (which may impair structural safety significantly), taking into account the fact that significant load events occur randomly in time with random intensities (Mori and Ellingwood 1993; Wang et al. 2015). Let $N$ be the number of load events that occur during the time interval $[0, T]$, at times $\widetilde{t}_{1}, \widetilde{t}_{2}, \ldots \widetilde{t}_{N}$, and $S\left(\widetilde{t}_{1}\right), S\left(\tilde{t}_{2}\right), \ldots S\left(\widetilde{t}_{N}\right)$ be the discrete load effects accordingly. With this, $\mathbb{L}(T)$ in Eq. (15) becomes

$$
\mathbb{L}(T)=\operatorname{Pr}\left\{R\left(\widetilde{t}_{1}\right)>S\left(\widetilde{t}_{1}\right) \cap \ldots \cap R\left(\widetilde{t}_{N}\right)>S\left(\widetilde{t}_{N}\right)\right\}
$$


If the load process is modeled as a stationary (homogeneous) Poisson process with an occurrence rate of $\lambda$, the probability mass function of $N$ is,

$$
\operatorname{Pr}(N=k)=\frac{(\lambda T)^{k}}{k !} \exp (-\lambda T), \quad k=0,1,2, \ldots
$$

With Eqs. (1), (2) and (17), subdividing each interval $\left[0, \tilde{t}_{j}\right]$ into $n_{j}$ identical sections $(j=1,2, \ldots N)$, the time-dependent reliability in Eq. (16) further becomes

$$
\mathbb{L}(T)=\operatorname{Pr}\left\{\bigcap_{j=1}^{N}\left[R_{0} \cdot\left(1-\sum_{i=1}^{n_{j}} \hat{d}\left(t_{i}\right) \cdot \epsilon\left(t_{i}\right)\right)>S\left(\tilde{t}_{j}\right)\right]\right\}
$$

Due to the difficulty of solving Eq. (18) numerically, a Monte Carlo simulation-based approach is used alternatively to estimate the time-dependent reliability. The procedure for each simulation run is summarized as follows.

1. Sample the initial resistance, denoted by $r_{0}$, according to the probability distribution function of $R_{0}$.

2. Simulate a Poisson variable $n$ with a mean value of $\lambda T$, and subsequently the samples of $n$ uniform variables within $[0, T]$, denoted by $\tilde{t}_{1}<\tilde{t}_{2}<\ldots<\widetilde{t}_{n}$.

3. For each $j=1,2, \ldots n$, subdivide the interval $\left[0, \tilde{t}_{j}\right]$ into $n_{j}$ identical sections, generate $\epsilon\left(t_{i}\right)$ for $i=1,2, \ldots n_{j}$ and compute $r\left(\tilde{t}_{j}\right)$ according to Eqs. (1) and (2).

4. Corresponding to $\widetilde{t}_{1}, \widetilde{t}_{2}, \ldots \widetilde{t}_{n}$, simulate $n$ independent load effects, $s\left(\widetilde{t}_{1}\right)$, $s\left(\tilde{t}_{2}\right), \ldots s\left(\tilde{t}_{n}\right)$.

5. If $s\left(\widetilde{t}_{j}\right)$ does not exceed $r\left(\tilde{t}_{j}\right)$ for all $j=1,2, \ldots n$, then the bridge is deemed as survival during $[0, T]$ and otherwise failure.

Performing the above procedure for $M$ times, if the structure survives for $m$ times, the time-dependent reliability is approximated by $m / M$ as $M$ is sufficiently large.

\section{Application of the proposed model in structural reliability assessment}

The applicability of the proposed resistance deterioration model in structural timedependent reliability assessment is demonstrated in this section. Consider a bridge subjected to the joint effect of live load $L$ and dead load $D$. The statistical information of structural resistance and load effects is summarized in Table 1. Two live load cases are considered, namely LL1 and LL2, with different coefficients of variation (COVs). The combination of nominal live load $L_{\mathrm{n}}$ and nominal dead load $D_{\mathrm{n}}$ is determined by $1.0 R_{\mathrm{n}}=1.25 D_{\mathrm{n}}+1.75 L_{\mathrm{n}}$ (AASHTO 2007), where $R_{\mathrm{n}}$ is the nominal initial resistance. The occurrence of live load is modeled by a homogeneous Poisson process with an occurrence rate of $\lambda$. It is assumed that $D_{\mathrm{n}}=L_{\mathrm{n}}$. It is noticed that the structural configuration considered herein is a simple representation and is for illustration purpose only; nonetheless it reflects the basic design concept of an engineered bridge. When the proposed deterioration method is applied to the reliability assessment of a real bridge, the determination of bridge's resistance would likely involve a finite element analysis, taking into account the bridge details such as structural type, span length, material strength, section geometry, among others.

Figure 2 plots the bridge's time-dependent failure probabilities subjected to LL1 for reference periods up to 40 years, using the deterioration model proposed in this paper. It is 
Table 1 Models of resistance and load

\begin{tabular}{lllll}
\hline & Mean & COV & Distribution & $\lambda\left(\mathrm{yr}^{-1}\right)$ \\
\hline Initial resistance & $1.05 R_{\mathrm{n}}$ & 0 & Deterministic & $/$ \\
Dead load & $1.00 D_{\mathrm{n}}$ & 0 & Deterministic & $/$ \\
Live load 1 (LL1) & $0.45 \mathrm{~L}_{\mathrm{n}}$ & 0.20 & Extreme Type I & 1.0 \\
Live load 2 (LL2) & $0.45 \mathrm{~L}_{\mathrm{n}}$ & 0.45 & Extreme Type I & 1.0 \\
\hline
\end{tabular}

assumed that $m=0.5, k=0.0018$ year, corresponding to the case that the deterioration function valued at the end of 40 years has a mean value of 0.7 , which is consistent with the scenario in Figs. 1a and b. Each $\epsilon\left(t_{i}\right)$ in Eq. (2) is assumed to follow a Gamma distribution. The failure probabilities are obtained by Monte Carlo simulation using 1,000,000 samples. The standard deviation of the deterioration function valued at the end of 40 years, $G(40)$, equals 0.14 in Fig. 2a and 0.21 in Fig. 2b. It is observed from Fig. 2 that a longer reference period results in a larger failure probability as expected, due to the accumulation of risks of structural failure. Furthermore, a greater value of $q$ leads to a smaller failure probability, and this effect is enhanced with a greater standard deviation of $G(40)$. This observation is consistent with that from Fig. 1c that a greater $q$ gives a smaller variation of the deterioration process. It is also seen from Fig. 2 that if $q<-0.5 m$, then using a Gamma deterioration model will underestimate the structural failure probability; on the other hand, the probability of failure will be overestimated by a Gamma model when $q>-0.5 m$.

The results in Fig. 2 are also informative of the bridge's service life. For example, in Fig. $2 \mathrm{a}$, with a target failure probability of 0.01 , the bridge's service life is estimated as 25 years with $q=-0.35$. This implies that the structural performance is not satisfactory beyond 25 years and thus needs relevant maintenance or repair measures. Furthermore, the choice of the deterioration model affects the accuracy of the service life prediction for the bridge. For example, in Fig. 2a, if the threshold for the failure probability is 0.01 , the bridge's service life is predicted to be 25 years, 27 years and 33 years respectively with $q=-0.35,-0.25$ and 1.5. The difference between the evaluations of bridge performance indicates the importance of properly selecting a resistance deterioration model.

The bridge's time-dependent failure probabilities considering the combination of dead load and LL2 are presented in Fig. 3, where the structural configuration is as in Fig. 2. It

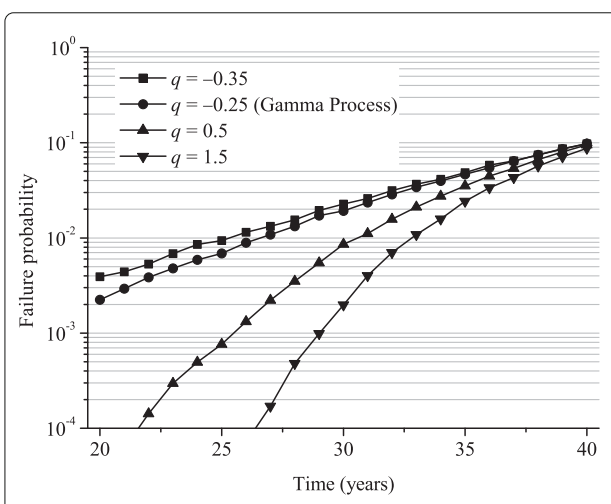

(a)

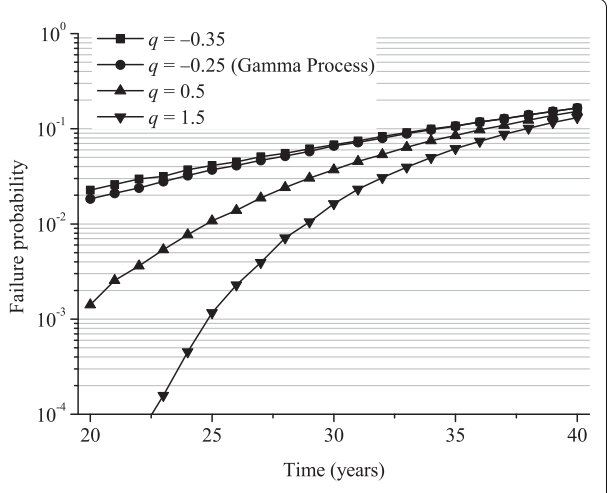

(b)

Fig. 2 Time-dependent failure probabilities for reference periods up to 40 years considering LL1. a $\sigma_{G(40)}=0.14 ; \mathbf{b} \sigma_{G(40)}=0.21$ 


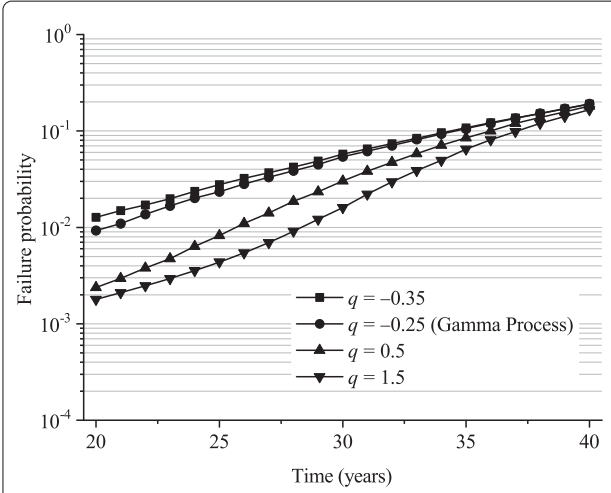

(a)

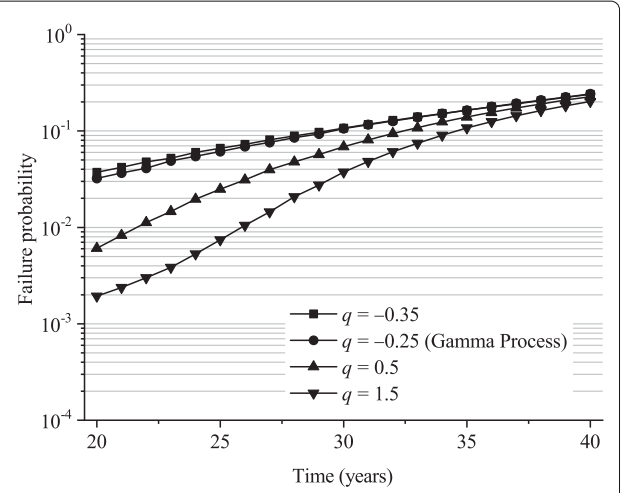

(b)

Fig. 3 As of Fig. 2 but considering LL2. $\mathbf{a} \sigma_{G(40)}=0.14 ; \mathbf{b} \sigma_{G(40)}=0.21$

can be seen that a greater variation of the live load results in a larger failure probability for the bridge. Correspondingly, with the same target reliability, the predicted service life is shortened by LL2. The impact of the deterioration model on structural failure probability, however, is similar to the observations in Fig. 2.

The dependence of structural failure probability on the occurrence rate of live load is presented in Fig. 4. The structural configuration is as in Fig. 2a except the value of $\lambda$. A greater live load occurrence rate leads to a larger failure probability. For both cases in Fig. 4 , when $\lambda \geq 0.6$, the structural failure probability increases approximately exponentially with respect to $\lambda$. The impact of $\lambda$ on the structural failure probability is weakened when a longer reference period is considered. This is because at the latter stage the structural reliability is dominated by the variation of the degraded resistance.

\section{Concluding remarks}

This paper has presented a new stochastic model for describing the resistance deterioration of aging bridges. The model is a modified version of the widely-used Gamma process model, releasing the dependence of variance and autocorrelation of the deterioration process on the mean value. This is achieved by introducing a new parameter that contributes to the time-variant variance of the deterioration process (c.f. the item $q$ in Eq. (8)). When

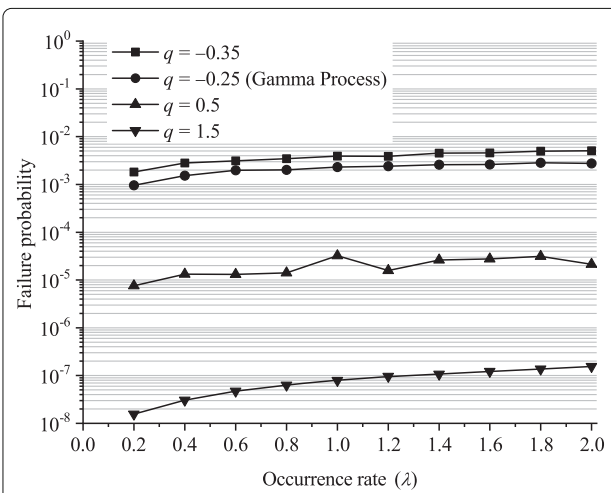

(a)

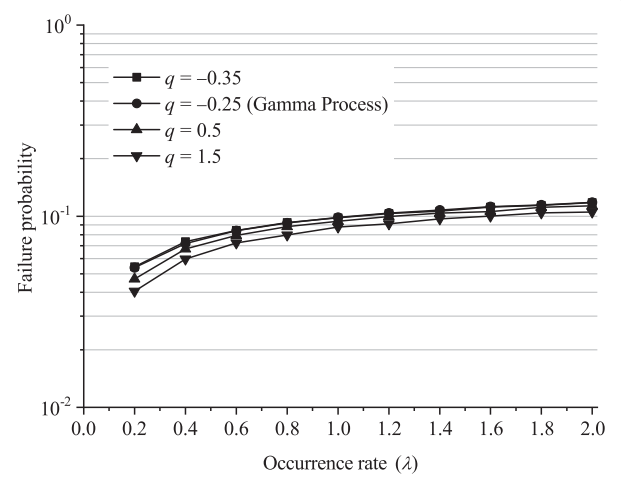

(b)

Fig. 4 Dependence of failure probability on the live load occurrence rate. $\mathbf{a} T=20$ years; $\mathbf{b} T=40$ years 
$q=-0.5 m$, where $m$ is the parameter reflecting the overall trend of the deterioration process in Eq. (7), then the proposed deterioration model degrades to a Gamma process. Analytical results show that the structural reliability is sensitive to the choice of resistance deterioration model. If $q<-0.5 m$, then using a Gamma deterioration model will overestimate structural reliability; otherwise, the structural reliability will be underestimated by a Gamma process-based deterioration model when $q>-0.5 m$. The proposed model is promising for use in time-dependent reliability assessment of aging bridges in the presence of resistance gradual deterioration.

Abbreviations

COV: Coefficient of variation

Acknowledgements

The author would like to acknowledge the thoughtful suggestions of two anonymous reviewers, which substantially improved the present paper.

Authors' information

C.W. received his PhD degree in June 2019 from the School of Civil Engineering, The University of Sydney, Australia. His research interests include natural hazards modelling and structural reliability analysis. Currently C.W. is a Research Fellow at the Department of Civil Engineering, Monash University, Australia. He is on the Early Career Editorial Board of the ASCE-ASME Journal of Risk and Uncertainty in Engineering Systems, Part A: Civil Engineering.

\section{Funding}

Not applicable.

Availability of data and materials

The datasets used and/or analysed during the current study are available from the corresponding author on reasonable request.

\section{Competing interests}

The author declares that he has no competing interests.

Received: 9 March 2020 Accepted: 26 May 2020

Published online: 07 July 2020

\section{References}

Mori Y, Ellingwood BR (1993) Reliability-based service-life assessment of aging concrete structures. J Struct Eng 119(5):1600-1621

Enright MP, Frangopol DM (1998) Service-life prediction of deteriorating concrete bridges. J Struct Eng 124(3):309-317

Stewart MG, Val DV (1999) Role of load history in reliability-based decision analysis of aging bridges. J Struct Eng 125(7):776-783

OBrien EJ, Bordallo-Ruiz A, Enright B (2014) Lifetime maximum load effects on short-span bridges subject to growing traffic volumes. Struct Saf 50:113-122

Dieulle L, Bérenguer C, Grall A, Roussignol M (2003) Sequential condition-based maintenance scheduling for a deteriorating system. Eur J Oper Res 150(2):451-461

Saassouh B, Dieulle L, Grall A (2007) Online maintenance policy for a deteriorating system with random change of mode. Reliab Eng Syst Saf 92(12):1677-1685

van Noortwijk JM, van der Weide JA, Kallen M-J, Pandey MD (2007) Gamma processes and peaks-over-threshold distributions for time-dependent reliability. Reliab Eng Syst Saf 92(12):1651-1658

Li Q, Wang C, Ellingwood BR (2015) Time-dependent reliability of aging structures in the presence of non-stationary loads and degradation. Struct Saf 52:132-141

Wang C, Li Q, Zou A, Zhang L (2015) A realistic resistance deterioration model for time-dependent reliability analysis of aging bridges. J Zhejiang Univ (Sci) A 16(7):513-524

Wang C, Feng K, Zhang L, Zou A (2019) Estimating the resistance of aging service-proven bridges with a Gamma process-based deterioration model. J Traffic Transp Eng (English Edition) 6(1):76-84

Kahle W, Mercier S, Paroissin C (2016) Degradation Processes in Reliability. Wiley, Hoboken. https://doi.org/10.1002/ 9781119307488

Billingsley P (1986) Probability and Measure. 2nd edn. John Wiley and Sons, New York

AASHTO (2007) LRFD Bridge Design Specifications. American Association of State Highway and Transportation Officials, Washington DC

\section{Publisher's Note}

Springer Nature remains neutral with regard to jurisdictional claims in published maps and institutional affiliations. 